\title{
Is the neutrophil-to-lymphocyte ratio a useful prognostic indicator in melanoma patients?
}

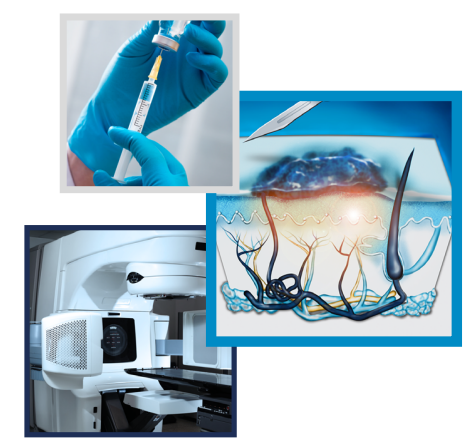

\author{
Joshua T Cohen ${ }^{1}$ (D), Thomas J Miner ${ }^{1}$ \& Michael P Vezeridis*,1 \\ ${ }^{1}$ Department of Surgery, Rhode Island Hospital, Warren Alpert Medical School of Brown University, Providence, RI, USA \\ *Author for correspondence: Michael_Vezeridis@brown.edu
}

\section{Practice points}

- Lymphocytes, namely natural killer cells and $\mathrm{CD}^{+}$cytotoxic $\mathrm{T}$ cells, are critical to the immune surveillance and destruction of nascent tumor cells and thus are antitumorigenic. Conversely neutrophils are largely protumorigenic, although an antitumorigenic subset likely does exist.

- Neutrophils expressing PD-L1 are able to induce T-cell apoptosis by binding to lymphocyte PD-1. Further, neutrophils can directly attenuate lymphocyte proliferation through production of reactive oxygen species and arginase-1.

- In localized advanced melanoma, a high neutrophil-to-lymphocyte ratio (NLR) is predictive of worse overall survival and disease-free survival. Paradoxically, there are limited data to suggest that in early stage localized melanoma a high NLR may be protective.

- For patients with metastatic melanoma, NLR is a useful prognostic marker for patients undergoing metastasectomy and for patients receiving immunotherapy.

- Future studies should focus on standardization of patient population and NLR cut-offs to improve external study validity. It is important to focus the outcomes of these studies on implementable management changes such as predicting sentinel or nonsentinel lymph node positivity, the need for adjuvant therapy and the response to immunotherapy.

The neutrophil-to-lymphocyte ratio (NLR) is gaining traction as a biomarker with utility in a variety of malignancies including melanoma. Intact lymphocyte function is necessary for tumor surveillance and destruction, and neutrophils play a role in suppressing lymphocyte proliferation and in the induction of lymphocyte apoptosis. Early research in melanoma indicates that in high-risk localized melanoma, a high NLR is correlated with worse overall and disease-free survival. Similarly, in metastatic melanoma treated with both metastasectomy and immunotherapies, an elevated NLR is predictive of shortened overall survival and progression-free survival. Future studies incorporating NLR into more traditional melanoma prognostic markers while employing more granular outcomes, are needed to realize the full potential of NLR.

First draft submitted: 1 May 2020; Accepted for publication: 25 June 2020; Published online:

25 August 2020

Keywords: immunotherapy • melanoma • neutrophil-to-lymphocyte ratio $\bullet$ prognosis

The neutrophil-to-lymphocyte ratio (NLR) is a readily available metric with an emerging role in the prognosis and management of a variety of solid tumors including breast, ovarian, colorectal, gastric, pancreatic, hepatocellular and neuroendocrine cancers [1-7]. Recently, a body of research has emerged employing NLR in the prognostication of both localized and metastatic melanoma [8-12].

This research is evolving during a period of rapid change in the management of melanoma. The first trial demonstrating the benefits of the immune checkpoint inhibitor ipilimumab in metastatic melanoma was published by Hodi et al. in 2010 [13]. Shortly thereafter, it was demonstrated that several other immunotherapies confer an overall survival benefit in melanoma patients [14-17]. The success of these systemic therapies allows for less nihilistic approach to advanced melanoma patients, with effective options for adjuvant or salvage therapies.

Subsequently, the Multicenter Selective Lymphadenectomy Trial II (MSLT-II) indicated that completion lymphadenectomy after a positive sentinel node biopsy (SLNB) did not improve disease specific survival, but that it 
provided prognostic information [18]. With these new data, the field must question if the benefits of sentinel node biopsy, namely the prognostic information, outweigh the risks associated with the operation given that a positive SLNB no longer changes operative management.

With these rapid changes in management and new therapeutic options, a number of questions arise. Can we predict who will have sentinel node or nonsentinel node metastasis? Is there a subset of patients that will benefit from completion lymph node dissection after a positive SLNB? Are there patients with localized disease that should receive adjuvant therapy? What patients with metastatic disease will respond to immunotherapy?

As the field develops, it will require novel markers of disease biology to better answer these questions and individualize care for patients with melanoma. Here we review the available literature on NLR in melanoma and discuss the future studies that will be necessary to further elucidate the role of NLR in the prognosis and management of melanoma.

\section{Role of the immune system in tumorigenesis}

While the recognition that the interchange between the innate and adaptive immune system as it relates to cancer prognosis is relatively new, the role of the immune system in both the initiation and prevention of tumorigenesis has long been established [19-21]. Significant gains have been made in the field of systemic treatments of various malignancies with the introduction of immunotherapies targeting lymphocyte function, specifically in the augmentation of the function of $\mathrm{CD}^{+}$cytotoxic $\mathrm{T}$ cells and natural killer (NK) cells by modulation of inhibitory checkpoint proteins [19,22]. Ipilimumab, an antibody against CTLA-4, was the first of these systemic immunotherapies that demonstrated efficacy in metastatic melanoma patients [13].

The success of these emerging therapies relies on the central role of the lymphocytes in the surveillance and destruction of cells that have undergone malignant transformation [19]. NK cells recognize and destroy cells lacking MHC-1, which should be present on all non-mutated nucleated cells and thus is the first line of lymphocyte defenses against tumorigenesis [23]. Following priming and activation, $\mathrm{CD}^{+}$cytotoxic $\mathrm{T}$ cells recognize and directly kill tumor cells through the release of perforin- and granzyme-containing granules $[19,24,25]$. CD $4^{+} \mathrm{Th} 1$ helper $\mathrm{T}$ cells act to support the role of cytotoxic $\mathrm{T}$ cells through the release of proinflammatory cytokines, namely IL-2, TNF- $\alpha$ and IFN- $\gamma[19,26-28]$. These lymphocyte-dependent processes are constantly working to identify and eradicate nascent tumor cells and, in fact, evading this lymphocyte surveillance is a key step in the development of tumorigenesis and metastasis $[19,23,24]$. Thus, it stands to reason that high levels of lymphocytes in the tumor infiltrate would correlate with improved outcomes from malignancies, which has been demonstrated to be true in melanoma [29].

Conversely, neutrophils are largely protumorigenic, and neutrophilia systemically and in the local tumor microenvironment has been linked to worse outcomes in a wide variety of malignancies including melanoma [30-32]. Neutrophils in isolation can promote metastasis. In a mouse model of hepatocellular carcinoma, neutrophil extracellular traps (NETs) have been demonstrated to support metastasis [33]. In this case, NETs are thought to improve tumor cell adhesion and promote extracellular matrix degradation, thereby allowing for enhanced tissue penetration at distant sites [33-35].

In addition to the direct protumorigenic nature of neutrophils, as exemplified by NETs, the effect of neutrophils on the T-cell response to malignancy also contributes to the protumorigenic environment [36-38]. Fridlender $e t a l$. have previously demonstrated in a mouse model of human mesothelioma that depletion of neutrophils from a tumor microenvironment causes an increase in the proportion of cytotoxic $\mathrm{T}$ cells in the tumor infiltrate, resulting in increased killing of malignant cells [39].

Neutrophils with a suppressive phenotype, and thus antitumorigenic, are released from bone marrow stores in response to non-resolving inflammatory stimuli (i.e., a tumor) [33]. These neutrophils are able to modulate the adaptive immune system by a multitude of mechanisms. Neutrophils can suppress T-cell proliferation by the release of hydrogen peroxide into immunologic synapses [34]. Additionally, suppression of T-cell proliferation is induced by neutrophils expressing arginase-1, which depletes the microenvironment of L-arginine, a conditionally essential amino acid necessary for T-cell receptor synthesis $[35,36]$. Finally, neutrophils can directly induce T-cell apoptosis by the interaction of neutrophil PD-L1 interacting with its receptor, PD-1 (Figure 1) [37,38,40]. Indeed, the ratio of neutrophil PD-L1 to lymphocyte PD-1 in the peritumoral environment has been demonstrated to be predictive of overall survival of hepatocellular carcinoma [41]. Taken together, the direct neutrophil modulation of T-cell effector 


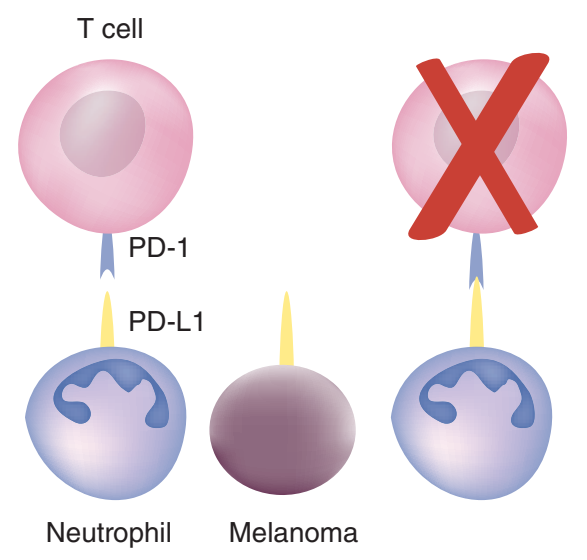

Figure 1. Neutrophil and tumor PD-L1 induce T-cell apoptosis.

\begin{tabular}{|c|c|c|c|c|c|c|}
\hline Study & NLR cut-off & Sample size & Patients & HR for death $(95 \% \mathrm{Cl})$ & $\begin{array}{l}\text { HR for melanoma specific death }(95 \% \\
\mathrm{CI})\end{array}$ & Ref. \\
\hline Davis et al. & 3 & 1,431 & $\mathrm{~T} 2 \mathrm{~b}+$ or $+\mathrm{SLNB}$ & - & $1.25(1.02-1.53)$ & [47] \\
\hline Ma et al. & 2.5 & 107 & Stage III & - & $3.82(1.26-11.56)$ & [11] \\
\hline Blakely et al. & 3 & 95 & $\geq 8 \mathrm{~mm}$ & $5.0(1.31-18.8)$ & NS & [9] \\
\hline Wade et al. & 2.5 & 1351 & All excisions w/SLNB & $0.45(0.43-0.50)$ & $0.53(0.45-0.625$ & [48] \\
\hline Lino-Silva et al. & 2 & 742 & All patients & $1.31(1.12-1.54)$ & - & [10] \\
\hline Ding et al. & $2-5$ & 3,207 & All patients & $2.23(1.64-3.04)$ & $2.19(1.78-2.69)$ & [51] \\
\hline
\end{tabular}

HR: Hazard ratio for the outcome if the NLR is greater than the cut-off value; NS: Not significant.

function combined with the central role of $\mathrm{T}$ cells in immune surveillance and destruction of tumor cells likely underlies the mechanism by which NLR has proven to be a useful prognostic marker.

\section{Confounding factors}

The utility of the NLR is limited by confounding factors such as systemic inflammation, infection and use of corticosteroids [42]. It is well established that acute bacterial infections drive emergency granulopoiesis, resulting in a relative increase in circulating neutrophils [43]. Further, the use of corticosteroids is known to increase neutrophil counts and decrease lymphocyte counts [42]. These confounding variables must be taken into account when interpreting the NLR on a patient by patient basis, as they may modulate the prognostic value.

\section{Localized melanoma}

Traditionally, tumor thickness, ulceration, mitotic index and SNLB positivity are the best prognostic indicators in localized melanoma [44-46]. However, as the care of cancer patients becomes increasingly individualized, finding additional prognostic markers will become increasingly important. This is especially true in patients with high-risk localized melanoma, where NLR is an area of interest (Table 1). In 2017, Davis et al. published a retrospective review of 1431 patients with high-risk melanoma that included T2b tumor or higher and patients with positive SLNB [47]. Utilizing an NLR ratio cut-off of 3, they demonstrated that high NLR was independently associated with worse melanoma specific mortality. High-NLR patients were significantly older, were more likely to be male, had thicker primary tumors, had higher mitotic indices and were more likely to have positive lymph nodes. Similarly, Ma et al. studied 107 patients with stage III melanoma [11]. They determined the optimal cut-off for NLR was 2.5. Patients with a low NLR had improved disease-free survival; however, these patients were more likely to have received systemic therapy, confounding the results. In addition, the authors of this review contributed to a retrospective review of 95 patients with ultrathick melanoma, defined as $\geq 8 \mathrm{~mm}$ depth of invasion [9]. Utilizing an a priori cut-off of 3, they found a high NLR was independently associated with improved overall survival (mean survival of 15 vs 29 months for high NLR and low NLR, respectively), but not progression-free survival. 
Broadening the scope, Wade et al. performed a multicenter cohort study investigating all patients that underwent excision for melanoma with an SLNB without evidence of distant metastasis over the course of 10 years [48]. They identified 1351 patients and determined an optimal cut-off for NLR of 2.5. Sixty-six percent of these patients were stage I. Patients with high NLR were more likely to have stage III disease, were more likely to be male, were significantly older, and were more likely have melanoma regression. Despite all of this, a high NLR was associated with better overall survival and disease-free survival. These data suggest that when looking at early stage low-risk melanoma there may be a paradoxical prognosis for high NLR. It could be hypothesized that in early stage disease a more antitumorigenic neutrophil is released into circulation and that in these patients a high NLR represents a proinflammatory antitumor environment. This would be consistent with data from mouse models of metastatic mesothelioma and lung cancer indicating that early stage tumors recruited more antitumorigenic neutrophils and late stage tumors recruited more protumorigenic neutrophils [49]. Previous data have also demonstrated that TGF$\beta$, the master regulator of pro- versus anti-tumorigenic neutrophil phenotype, is dependent on Notch1, which is elevated to a greater degree in advanced melanoma [50]. In this way early melanoma may have lower TGF- $\beta$ signaling and thus more antitumorigenic neutrophils. Additional studies at the cellular and molecular level will be needed to understand the nuance of these potentially complex interactions.

Utilizing a more comprehensive patient cohort Lino-Silva et al. performed a retrospective review of all patients treated for melanoma over the 10-year study period [10]. They identified 742 patients and determined an optimal NLR cut-off of 2 . They found that a high NLR predicted decreased overall survival. These outcomes were largely driven by stage II patients, which was the only stage found to have a significant change in median overall survival time in subgroup analysis. Patients with a high NLR were significantly more likely to have a lymph node metastasis which was not specifically addressed in multivariate analysis, although clinical stage was included. However, it could be argued that NLR and lymph node positivity are not independent variables. Robinson $e$ al. published a multicenter cohort study of 1489 stage I-III locoregional melanoma patients and found that and NLR $\geq 2.3$ was correlated with a $30 \%$ increased risk of micrometastasis on SLNB [12].

Finally, a meta-analysis including 12 studies and 3207 stage I-IV patients found a significantly worse disease free- and overall-survival in patients with high NLR as set by individual studies [51].

Taken together, these data indicate that in advanced nonmetastatic melanoma, NLR likely offers prognostic insight into overall survival and melanoma recurrence. At this point, however, the optimal cut-off for NLR is unknown, with investigators reporting values between 2 and 5 [9,10,47,48,51]. Further, the directionality of effect is inconsistent, with data indicating that in early stage melanoma a high NLR may be protective [48]. These differences are most likely attributable to the inclusion criteria of a given study and the resulting heterogeneity of the study populations. Looking forward, it is imperative to define which patients with localized melanoma require additional prognostic information and delineate either a standardized NLR cutoff or treat NLR as a continuous variable to improve external validity.

Future studies should focus on the ability of NLR to predict SLNB positivity or nonsentinel node metastasis with hope of clarifying operative indications on the nodal basins. Specifically, NLR, in combination with traditional biopsy-driven prognostic factors, may identify a low-risk patient population in whom SLNB does not add additional prognostic information. The converse may also be true in that there may be a patient so high risk of nonsentinel node metastasis and regional recurrence that a completion lymphadenectomy would be warranted.

There may be utility in moving beyond studying the peripheral blood NLR and investigating the peritumoral and intratumoral NLR, which is known to be predictive of overall survival in hepatocellular carcinoma [41]. Translating these findings to melanoma, however, may prove challenging. While peripheral blood NLR is readily available and easy to analyze, identifying the relatively rare neutrophils in the tumor microenvironment is labor intensive by immunohistochemistry and there are technical limitations to the use of flow cytometry in melanoma samples. Beyond a simple ratio, it may be possible to offer improved granularity by differentiating protumorigenic from antitumorigenic neutrophils in the local tumor microenvironment, which may indicate a switch from low-risk to high-risk tumor $[49,52,53]$. Neutrophil arginase-1 release is one potential marker of this transition as it has been shown to attenuate the lymphocyte response in a mouse model of renal cell carcinoma [36,54]. Other potential targets could include the degree of reactive oxygen species production [33,34,55] and level of PD-L1 expression [41].

\section{Metastatic melanoma}

The prognostic value of NLR extends beyond localized disease to patients with distant metastasis (Table 2). Two studies have investigated the role of NLR in the prognosis of patients who underwent metastasectomy for Stage 


\begin{tabular}{|c|c|c|c|c|c|c|c|}
\hline Study & NLR cut-off & Therapy & Sample size & Patients & HR for death $(95 \% \mathrm{Cl})$ & $\begin{array}{l}\text { HR for melanoma } \\
\text { progression }(95 \% \mathrm{Cl})\end{array}$ & Ref. \\
\hline Kanastsios et al. & 5 & Metastasectomy & 95 & Stage IV & 3.59 [NOT PUBLISHED] & - & [56] \\
\hline Cananzi et al. & 5 & Metastasectomy & 77 & Stage IV & $6.98(2.07-23.60)$ & - & [8] \\
\hline Teterycz et al. & 4 & $\begin{array}{l}\text { BRAF and MEK } \\
\text { inhibitors }\end{array}$ & 215 & Stage IV-BRAF+ & NS & $1.81(1.16-2.85)$ & [60] \\
\hline $\begin{array}{l}\text { Cocorocchio } \\
\text { et al. }\end{array}$ & 5 & $\begin{array}{l}\text { BRAF and MEK } \\
\text { inhibitors }\end{array}$ & 90 & Stage IV-BRAF+ & $2.04(1.18-3.57)$ & $2.17(1.32-4.35)$ & [58] \\
\hline Finon et al. & 4 & BRAF inhibitors & 49 & $\begin{array}{l}\text { Unresectable stage III and stage } \\
\text { IV }\end{array}$ & - & $3.24(1.30-8.12)$ & [59] \\
\hline Bartlett et al. & 5 & PD-1 inhibitors & 224 & Stage IV & $2.0(1.3-2.9)$ & $1.7(1.2-2.4)$ & [61] \\
\hline Cassidy et al. & 5 & Ipilimumab & 197 & $\begin{array}{l}\text { Unresectable stage III and stage } \\
\text { IV }\end{array}$ & $2.03(1.49-2.77)$ & $1.81(1.33,2.45)$ & [62] \\
\hline Zaragoza et al. & 4 & Ipilimumab & 58 & $\begin{array}{l}\text { Unresectable stage III and stage } \\
\text { IV }\end{array}$ & $2.2(1.01-4.78)$ & - & [63] \\
\hline Fujisawa et al. & 2.2 & Nivolumab & 90 & $\begin{array}{l}\text { Unresectable stage III and stage } \\
\text { IV }\end{array}$ & - & 4.16 [NOT PUBLISHED] & [64] \\
\hline Ferrucci et al. & 3 & Ipilimumab & 855 & $\begin{array}{l}\text { Unresectable stage III and stage } \\
\text { IV }\end{array}$ & $5.76(4.29-7.75)$ & $4.10(30.8-5.46)$ & [65] \\
\hline Rosner et al. & 4.73 & $\begin{array}{l}\text { Nivolumab and } \\
\text { Ipilimumab }\end{array}$ & 209 & $\begin{array}{l}\text { Unresectable stage III and stage } \\
\text { IV }\end{array}$ & $1.95(1.11-.43)$ & - & [66] \\
\hline
\end{tabular}

HR: Hazard ratio for the outcome if the NLR is greater than the cut-off value; NS: Not significant.

IV melanoma [8,56]. Both studies utilized an a priori cut-off of 5 based on previously published work. Kanatsios et al. studied 95 patients who underwent definitive resection of metastatic melanoma [56]. An R0 resection was achieved in $93 \%$ of patients and $32 \%$ of patients had no further disease progression during the study period. Patients with an NLR $>5$ had significantly worse median overall survival at 15 months compared with 65 months in the low-NLR cohort. Canazi et al. focused on 44 patients that underwent metastasectomy for intraabdominal melanoma metastasis [8]. In this study, only $43.2 \%$ of patients achieved complete cytoreduction. An NLR of over 5 was independently predictive of mortality in the entire study group as well as on subgroup analysis of patients that achieved complete cytoreduction.

Perhaps the most cutting-edge use of NLR in melanoma patients is in predicting, which patients will respond to targeted therapies [57-59]. Teterycz et al. investigated 215 patients with metastatic or inoperable BRAF-positive melanoma that were treated with combination BRAF and MEK inhibitors, either dabrafenib with trametinib or vemurafenib with cobimetinib $[58,60]$. They determined that an NLR of greater than 4 was independently associated with diminished progression-free survival. Median progression-free survival was 10.5 months. The investigators also treated NLR as a continuous variable and found an independent association of NLR and disease progression with a hazard ratio of 1.06 (95\% CI: 1.02-1.11) per unit NLR change.

Similarly, several studies have demonstrated the prognostic benefits of NLR in patients receiving checkpoint inhibitors [61-66]. Bartlett et al. studied 224 patients with stage IV melanoma undergoing systemic PD-1 inhibitor monotherapy [61]. Seventy-nine percent of patients underwent treatment with pembrolizumab, while the remaining received nivolumab. Employing an a priori cut off of 5, there was an independent association between high NLR and both shortened overall survival and shortened time to disease progression. Patients in the high NLR group were more likely to have a greater disease burden and poorer overall performance status. Interestingly, an NLR increase of $\geq 30 \%$ after initiation of therapy was also associated with shortened overall survival at 13.5 months in the NLR cohort with $\mathrm{a} \geq 30 \%$ compared with 47 months in patients with $<30 \%$ rise.

Two studies have investigated the role of NLR in patients being treated with the anti-CTLA-4 antibody, ipilimumab [62,63]. The larger of the two studied 197 patients with unresectable stage III or stage IV melanoma [62]. They found that baseline NLR as well as NLR at week 3, week 6, and week 9 after initiation of treatment were independently correlated with both disease-free and progression-free survival utilizing a cut-off of 5 . Here again, $\mathrm{a} \geq 30 \%$ rise in NLR correlates with worse progression free survival and overall survival. This study included a small cohort (65 patients) that received BRAF inhibitors. They found baseline NLR was independently associated with overall- and progression-free survival at 12 months. Similar results were found in a study of 58 patients who received ipilimumab, utilizing a lower cutoff of 4 [63]. In this study, NLR was also treated as a continuous variable 
and again was independently associated with overall survival with a hazard ratio of 1.10 (95\% CI: $1.01-1.19)$ per unit increase.

As novel targets and immunotherapies are developed, and new combination systemic regimens are established, choosing the optimal treatment strategy for patients with metastatic melanoma will become increasingly complex [6769]. As more options become available, the early identification of treatment success and, perhaps more importantly, treatment failure will become increasingly important. In this context, NLR holds the potential to predict the success of treatment prior to initiation and then again after therapy has started. Of particular interest would be utilizing NLR as a continuous variable in assessing risk of treatment failure [60,63]. Ideally, NLR along with other known predictors of melanoma survival could be combined into a nomogram to help predict survival while on a given therapy. This strategy has been successful in predicting overall survival from hepatocellular carcinoma [70]. Further investigation into the dynamics of NLR modulation after initiation of systemic therapies could result in more granular and timely indicators of response to therapy [61-63]. Beyond the patients with stage IV disease, NLR may hold promise in identifying patients with localized disease that are at high risk for regional or distant recurrence and thus could benefit from adjuvant immunotherapy [61-63].

\section{Future perspective}

The full utility of NLR in the prognosis and management of melanoma has not yet been realized but early results are promising. The preliminary data indicate that a high NLR is a useful prognostic marker in high-risk nonmetastatic melanoma, although the cut-offs in this field are variable and the patient populations remain murky. In patients with unresectable disease, NLR is good marker for progression free and overall survival. Ideally, research will shift to viewing NLR as a continuous variable in combination with other known prognostic indicators to provide a more holistic approach to prognostication. Further characterization of neutrophil subsets with the understanding that not all neutrophils are created equally may aid in improving precision. Utilizing this more complete dataset may allow for prediction of more granular outcomes like SLNB positivity, nonsentinel node metastasis, need for adjuvant therapy, and response to immunotherapies. Taken together, incorporating NLR into the prognostic algorithms for melanoma holds the potential to offer more individualized and comprehensive cancer care.

Financial \& competing interests disclosure

The authors have no relevant affiliations or financial involvement with any organization or entity with a financial interest in or financial conflict with the subject matter or materials discussed in the manuscript. This includes employment, consultancies, honoraria, stock ownership or options, expert testimony, grants or patents received or pending, or royalties.

No writing assistance was utilized in the production of this manuscript.

Open access

This work is licensed under the Attribution-NonCommercial-NoDerivatives 4.0 Unported License. To view a copy of this license, visit http://creativecommons.org/licenses/by-nc-nd/4.0/

\section{References}

1. Cao L-L, Lu J, Lin J-X et al. Nomogram based on tumor-associated neutrophil-to-lymphocyte ratio to predict survival of patients with gastric neuroendocrine neoplasms. World J. Gastroenterol. 23(47), 8376-8386 (2017).

2. Chen G, Zhu L, Yang Y, Long Y, Li X, Wang Y. Prognostic role of neutrophil to lymphocyte ratio in ovarian cancer: a meta-analysis. Technol. Cancer Res. T. 17, 1533033818791500 (2018).

3. Ethier J-L, Desautels D, Templeton A, Shah PS, Amir E. Prognostic role of neutrophil-to-lymphocyte ratio in breast cancer: a systematic review and meta-analysis. Breast Cancer Res. 19(1), 2 (2017).

4. Templeton AJ, McNamara MG, Šeruga B et al. Prognostic role of neutrophil-to-lymphocyte ratio in solid tumors: a systematic review and meta-analysis. J. National Cancer Inst. 106(6), dju124 (2014).

5. Szor DJ, Dias AR, Pereira MA et al. Prognostic role of neutrophil/lymphocyte ratio in resected gastric cancer: a systematic review and meta-analysis. Clinics 73, e360 (2018).

6. Santos TD, Jammal MP, Silveira TP, Murta EFC, Nomelini RS. Stromal IL2 is related to the neutrophil/lymphocyte ratio in epithelial ovarian cancer. Pathologica 111(2), 62-66 (2019).

7. Liu K, Zhao K, Wang L, Sun E. The prognostic values of tumor-infiltrating neutrophils, lymphocytes and neutrophil/lymphocyte rates in bladder urothelial cancer. Pathol. Res. Pract. 214(8), 1074-1080 (2018).

8. Cananzi FCM, Dalgleish A, Mudan S. Surgical management of intraabdominal metastases from melanoma: role of the neutrophil to lymphocyte ratio as a potential prognostic factor. World J. Surg. 38(6), 1542-1550 (2014). 
9. Blakely AM, Cohen JT, Comissiong DS, Vezeridis MP, Miner TJ. Prognosis and management of thick and ultrathick melanoma. Am. J. Clin. Oncol. 42(11), 824-829 (2019).

10. Lino-Silva LS, Salcedo-Hernández RA, García-Pérez L, Meneses-García A, Zepeda-Najar C. Basal neutrophil-to-lymphocyte ratio is associated with overall survival in melanoma. Melanoma Res. 27(2), 140-144 (2017).

11. Ma J, Kuzman J, Ray A et al. Neutrophil-to-lymphocyte Ratio (NLR) as a predictor for recurrence in patients with stage III melanoma. Sci. Rep. 8(1), 4044 (2018).

12. Robinson AV, Keeble C, Lo MCI et al. The neutrophil-lymphocyte ratio and locoregional melanoma: a multicentre cohort study. Cancer Immunol. Immunother. 69(4), 559-568 (2020).

13. Hodi FS, O’Day SJ, McDermott DF et al. Improved survival with ipilimumab in patients with metastatic melanoma. New Engl. J. Med. 363(8), 711-723 (2010).

14. Larkin J, Chiarion-Sileni V, Gonzalez R et al. Five-year survival with combined nivolumab and ipilimumab in advanced melanoma. New Engl. J. Med. 381(16), 1535-1546 (2019).

15. Eggermont AMM, Blank CU, Mandala M et al. Adjuvant pembrolizumab versus placebo in resected stage iii melanoma. New Engl. J. Med. 378(19), 1789-1801 (2018).

16. Schachter J, Ribas A, Long GV et al. Pembrolizumab versus ipilimumab for advanced melanoma: final overall survival results of a multicentre, randomised, open-label phase 3 study (KEYNOTE-006). Lancet 390(10105), 1853-1862 (2017).

17. Mackiewicz J, Mackiewicz A. BRAF and MEK inhibitors in the era of immunotherapy in melanoma patients. Contemp. Oncol. (Pozn) 2018(1), 68-72 (2018).

18. Faries MB, Thompson JF, Cochran AJ et al. Completion dissection or observation for sentinel-node metastasis in melanoma. N. Engl. J. Med. 376(23), 2211-2222 (2017).

19. Gonzalez H, Hagerling C, Werb Z. Roles of the immune system in cancer: from tumor initiation to metastatic progression. Gene Dev. 32(19-20), 1267-1284 (2018).

20. Vahidian F, Duijf PHG, Safarzadeh E, Derakhshani A, Baghbanzadeh A, Baradaran B. Interactions between cancer stem cells, immune system and some environmental components: friends or foes? Immunol. Lett. 208, 19-29 (2019).

21. Khalil DN, Budhu S, Gasmi B et al. Chapter one the new era of cancer immunotherapy manipulating t-cell activity to overcome malignancy. Adv. Cancer Res. 128, 1-68 (2015).

22. Souza-Fonseca-Guimaraes F, Cursons J, Huntington ND. The emergence of natural killer cells as a major target in cancer immunotherapy. Trends Immunol. 40, 142-158 (2019).

23. Iannello A, Thompson TW, Ardolino M, Marcus A, Raulet DH. Immunosurveillance and immunotherapy of tumors by innate immune cells. Curr. Opin. Immunol. 38, 52-58 (2016).

24. Matsushita H, Vesely MD, Koboldt DC et al. Cancer exome analysis reveals a T-cell-dependent mechanism of cancer immunoediting. Nature 482(7385), 400-404 (2012).

25. Hanson HL, Donermeyer DL, Ikeda $\mathrm{H}$ et al. Eradication of established tumors by CD8+ $\mathrm{T}$ cell adoptive immunotherapy. Immunity 13(2), 265-276 (2000).

26. Pardoll DM, Topalian SL. The role of CD4+ T cell responses in antitumor immunity. Curr. Opin. Immunol. 10(5), 588-594 (1998).

27. Kalams SA, Walker BD. The critical need for CD4 Help in maintaining effective cytotoxic T lymphocyte responses. J. Exp. Med. 188(12), 2199-2204 (1998).

28. Shankaran V, Ikeda $\mathrm{H}$, Bruce AT et al. IFN $\gamma$ and lymphocytes prevent primary tumour development and shape tumour immunogenicity. Nature 410(6832), 1107-1111 (2001).

29. Clemente CG, Mihm MC, Bufalino R, Zurrida S, Collini P, Cascinelli N. Prognostic value of tumor infiltrating lymphocytes in the vertical growth phase of primary cutaneous melanoma. Cancer 77(7), 1303-1310 (1996).

30. Donskov F. Immunomonitoring and prognostic relevance of neutrophils in clinical trials. Semin. Cancer Biol. 23(3), $200-207$ (2013).

31. Jensen TO, Schmidt H, Møller HJ et al. Intratumoral neutrophils and plasmacytoid dendritic cells indicate poor prognosis and are associated with pSTAT3 expression in AJCC stage I/II melanoma. Cancer 118(9), 2476-2485 (2011).

32. Shaul ME, Fridlender ZG. Neutrophils as active regulators of the immune system in the tumor microenvironment. J. Leukocyte Biol. 102(2), 343-349 (2017).

33. Leliefeld PHC, Wessels CM, Leenen LPH, Koenderman L, Pillay J. The role of neutrophils in immune dysfunction during severe inflammation. Critical Care. 20(1), 1-9 (2016).

34. Pillay J, Kamp VM, van Hoffen E et al. A subset of neutrophils in human systemic inflammation inhibits $\mathrm{T}$ cell responses through Mac-1. J. Clin. Invest. 122(1), 327-336 (2012).

35. Darcy CJ, Minigo G, Piera KA et al. Neutrophils with myeloid derived suppressor function deplete arginine and constrain $\mathrm{T}$ cell function in septic shock patients. Crit. Care 18(4), R163 (2014). 
36. Zea AH, Rodriguez PC, Atkins MB et al. Arginase-producing myeloid suppressor cells in renal cell carcinoma patients: a mechanism of tumor evasion. Cancer Res. 65(8), 3044-3048 (2005).

37. de Kleijn S, Langereis JD, Leentjens J et al. IFN- $\gamma$-stimulated neutrophils suppress lymphocyte proliferation through expression of PD-L1. PLoS ONE 8(8), e72249 (2013).

38. Langereis JD, Pickkers P, de Kleijn S, Gerretsen J, de MI Jonge, Kox M. Spleen-derived IFN- $\gamma$ induces generation of PD-L1+-suppressive neutrophils during endotoxemia. J. Leukoc. Biol. 102(6), 1401-1409 (2017).

39. Fridlender ZG, Sun J, Kim S et al. Polarization of tumor-associated neutrophil phenotype by TGF- $\beta$ : "N1" versus "N2" TAN. Cancer Cell 16(3), 183-194 (2009).

40. Wang J-F, Li J-B, Zhao Y-J et al. Up-regulation of programmed cell death 1 ligand 1 on neutrophils may be involved in sepsis-induced immunosuppression: an animal study and a prospective case-control study. Anesthesiology 122(4), 852-863 (2015).

41. He G, Zhang H, Zhou J et al. Peritumoural neutrophils negatively regulate adaptive immunity via the PD-L1/PD-1 signalling pathway in hepatocellular carcinoma. J. Exp. Clin. Cancer Res. 34(1), 141 (2015).

42. Karakonstantis S, Kalemaki D, Tzagkarakis E, Lydakis C. Pitfalls in studies of eosinopenia and neutrophil-to-lymphocyte count ratio. Infect. Dis. 50(3), 163-174 (2017).

43. Manz MG, Boettcher S. Emergency granulopoiesis. Nat. Rev. Immunol. 14(5), 302-314 (2014).

44. Amin MB, Greene FL, Edge SB et al. The Eighth Edition AJCC Cancer Staging Manual: continuing to build a bridge from a population-based to a more "personalized" approach to cancer staging. CA Cancer J. Clin. 67(2), 93-99 (2017).

45. Gershenwald JE, Scolyer RA, Hess KR et al. Melanoma staging: evidence-based changes in the American Joint Committee on Cancer eighth edition cancer staging manual. CA Cancer J. Clin. 67(6), 472-492 (2017).

46. Bartlett EK, Karakousis GC. Current staging and prognostic factors in melanoma. Surg. Oncol. Clin. N. Am. 24(2), 215-227 (2015).

47. Davis JL, Langan RC, Panageas KS et al. Elevated blood neutrophil-to-lymphocyte ratio: a readily available biomarker associated with death due to disease in high risk nonmetastatic melanoma. Ann. Surg. Oncol. 24(7), 1989-1996 (2017).

48. Wade RG, Robinson AV, Lo MCI et al. Baseline neutrophil-lymphocyte and platelet-lymphocyte ratios as biomarkers of survival in cutaneous melanoma: a multicenter cohort study. Ann. Surg. Oncol. 25(11), 3341-3349 (2018).

49. Mishalian I, Bayuh R, Levy L, Zolotarov L, Michaeli J, Fridlender ZG. Tumor-associated neutrophils (TAN) develop pro-tumorigenic properties during tumor progression. Cancer Immunol. Immunother. 62(11), 1745-1756 (2013).

50. Yang Z, Qi Y, Lai $\mathrm{N}$ et al. Notch1 signaling in melanoma cells promoted tumor-induced immunosuppression via upregulation of TGF-ß1. J. Exp. Clin. Cancer. Res. 37(1), 1 (2018).

51. Ding Y, Zhang S, Qiao J. Prognostic value of neutrophil-to-lymphocyte ratio in melanoma. Medicine 97(30), e11446-11447 (2018).

52. Sionov RV, Fridlender ZG, Granot Z. The multifaceted roles neutrophils play in the tumor microenvironment. Cancer Microenviron. 8(3), 125-158 (2015).

53. Bonavita O, Massara M, Bonecchi R. Chemokine regulation of neutrophil function in tumors. Cytokine Growth Factor R. 30, 81-86 (2016).

54. Rotondo R, Barisione G, Mastracci L et al. IL-8 induces exocytosis of arginase 1 by neutrophil polymorphonuclears in nonsmall cell lung cancer. Int. J. Cancer 125(4), 887-893 (2009).

55. Pillay J, Tak T, Kamp VM, Koenderman L. Immune suppression by neutrophils and granulocytic myeloid-derived suppressor cells: similarities and differences. Cell. Mol. Life Sci. 70(20), 3813-3827 (2013).

56. Kanatsios S, Project MM, Suen CSNLW, Cebon JS, Gyorki DE. Neutrophil to lymphocyte ratio is an independent predictor of outcome for patients undergoing definitive resection for stage IV melanoma. J. Surg. Oncol. 118(6), 915-921 (2018).

57. Sacdalan DB, Lucero JA, Sacdalan DL. Prognostic utility of baseline neutrophil-to-lymphocyte ratio in patients receiving immune checkpoint inhibitors: a review and meta-analysis. Oncotargets Ther. 11, 955-965 (2018).

58. Cocorocchio E, Martinoli C, Gandini S et al. Baseline neutrophil-to-lymphocyte ratio (NLR) is associated with outcome of patients treated with BRAF inhibitors. Clin. Transl, Oncol. doi: 10.1007/s12094-020-02320-y (2020).

59. Finon A, Zaragoza J, Maillard $\mathrm{H}$ et al. A high neutrophil to lymphocyte ratio prior to BRAF inhibitor treatment is a predictor of poor progression-free survival in patients with metastatic melanoma. Eur. J. Dermatol. 28(1), 38-43 (2018).

60. Teterycz P, Jagodziska-Mucha P, Cybulska-Stopa B et al. High baseline neutrophil-to-lymphocyte ratio predicts worse outcome in patients with metastatic BRAF-positive melanoma treated with BRAF and MEK inhibitors. Melanoma Res. 28(5), 435-441 (2018).

61. Bartlett EK, Flynn JR, Panageas KS et al. High neutrophil-to-lymphocyte ratio (NLR) is associated with treatment failure and death in patients who have melanoma treated with PD-1 inhibitor monotherapy. Cancer 126(1), 76-85 (2020).

62. Cassidy MR, Wolchok RE, Zheng J et al. Neutrophil to lymphocyte ratio is associated with outcome during ipilimumab treatment. EBiomedicine 18, 56-61 (2017).

63. Zaragoza J, Caille A, Beneton $\mathrm{N}$ et al. High neutrophil to lymphocyte ratio measured before starting ipilimumab treatment is associated with reduced overall survival in patients with melanoma. Brit. J. Dermatol. 174(1), 146-151 (2016) 
64. Fujisawa Y, Yoshino K, Otsuka A et al. Baseline neutrophil to lymphocyte ratio combined with serum lactate dehydrogenase level associated with outcome of nivolumab immunotherapy in a Japanese advanced melanoma population. Brit. J. Dermatol. 179(1), 213-215 (2018).

65. Ferrucci PF, Ascierto PA, Pigozzo J et al. Baseline neutrophils and derived neutrophil-to-lymphocyte ratio: prognostic relevance in metastatic melanoma patients receiving ipilimumab. Ann. Oncol. 27(4), 732-738 (2017).

66. Rosner S, Kwong E, Shoushtari AN et al. Peripheral blood clinical laboratory variables associated with outcomes following combination nivolumab and ipilimumab immunotherapy in melanoma. Cancer Med. 7(3), 690-697 (2018).

67. Mattia G, Puglisi R, Ascione B, Malorni W, Carè A, Matarrese P. Cell death-based treatments of melanoma:conventional treatments and new therapeutic strategies. Cell Death Dis. 9(2), 112 (2018).

68. Mandalà M, Rutkowski P. Rational combination of cancer immunotherapy in melanoma. Virchows Arch. 474(4), $433-447$ (2019).

69. Kuklinski LF, Yan S, Li Z et al. VISTA expression on tumor-infiltrating inflammatory cells in primary cutaneous melanoma correlates with poor disease-specific survival. Cancer Immunol. Immunother. 67(7), 1113-1121 (2018).

70. Yuan J, Liang H, Li J et al. Peripheral blood neutrophil count as a prognostic factor for patients with hepatocellular carcinoma treated with sorafenib. Mol. Clin. Oncol. 7(5), 837-842 (2017). 
